\title{
Symptomatic Liver Metastasis Prompting Diagnosis of Uveal Melanoma
}

\author{
Caroline C. Awh Matthew W. Wilson \\ Department of Ophthalmology, Hamilton Eye Institute, University of Tennessee Health Science Center, College of \\ Medicine, Memphis, TN, USA
}

\section{Established Facts}

- Uveal melanoma metastasizes in up to $50 \%$ of the cases, most frequently to the liver, but it is exceptionally rare for metastasis to occur prior to primary tumor diagnosis.

- Cytogenetic analysis is available to identify high-risk tumors and calculate estimated rates of survival.

\section{Novel Insights}

- This is the first reported case of symptomatic liver metastasis as the presenting sign of metastatic uveal melanoma.

- Magnetic resonance imaging can detect uveal melanoma but is insufficient to rule out primary uveal melanoma when the source of metastatic lesions are unknown.

- If the primary tumor is unknown, cytogenetic analysis can be used to confirm a diagnosis of uveal melanoma in addition to risk stratification.

\section{Keywords}

Uveal melanoma - Malignant melanoma .

Cytogenetics of uveal melanoma uveal melanoma. This was ultimately confirmed to be the primary site of his malignant metastatic melanoma, which caused his death within 2 months of presentation.

(c) 2019 S. Karger AG, Basel

\begin{abstract}
We report the case of a 66-year-old male with symptomatic liver metastasis as the presenting manifestation of primary uveal melanoma. Upon initial presentation to an emergency department with 2 months of abdominal pain, back pain, and unintended weight loss, a computed tomography scan demonstrated diffuse liver and bone lesions, prompting referral to a medical oncologist. He was eventually examined by an ophthalmologist, who found a lesion suspicious for
\end{abstract}

(c) 2019 S. Karger AG, Basel

\section{Introduction}

We report the first documented case of symptomatic liver metastasis as the presenting sign of metastatic uveal melanoma. Seventy percent of the patients with uveal melanoma are diagnosed after they present with ophthalmic complaints, and the majority of the remaining cases are detected incidentally on eye examination. Fewer than

\section{KARGER}

karger@karger.com

www.karger.com/oop
Matthew W. Wilson, MD, FACS

University of Tennessee Health Science Center

930 Madison Ave, Room 476

Memphis, TN 38163 (USA)

E-Mail mwilson5@uthsc.edu 


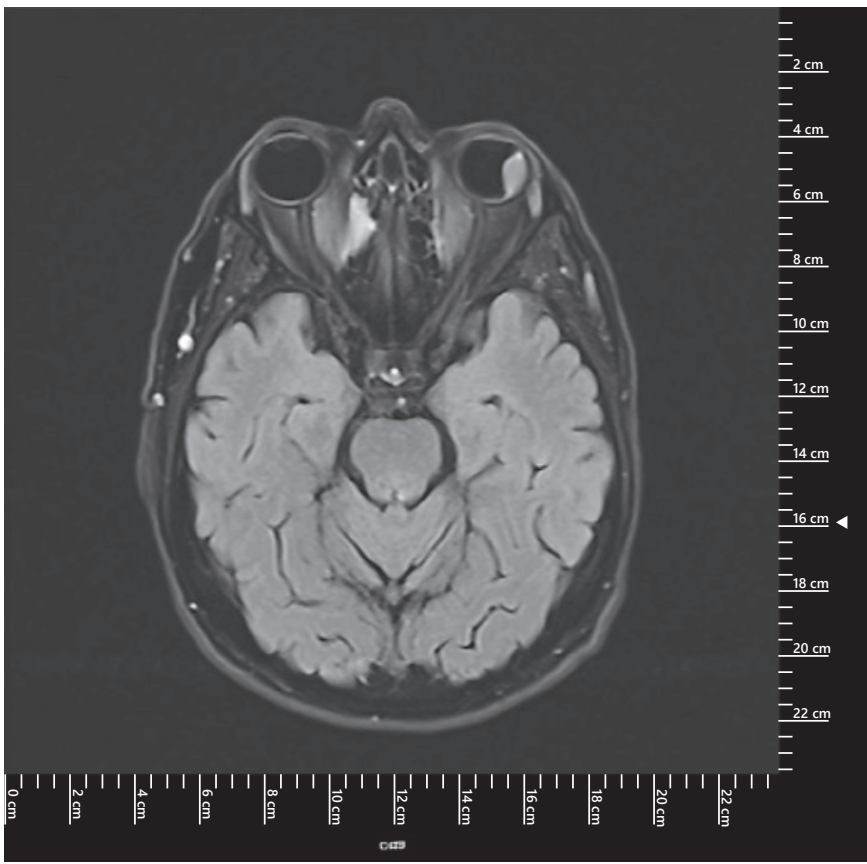

Fig. 1. MRI of the orbits depicting an $11 \times 7 \mathrm{~mm}$ enhancing nodule along the posterolateral aspect of the left globe.

$4 \%$ of the patients have detectable metastatic disease at the time of their diagnosis. The development of liver metastasis with an unknown primary tumor should raise clinical suspicion for uveal melanoma and prompt a thorough ophthalmologic workup.

\section{Case Report and Case Presentation}

A 66-year-old Caucasian male presented to an outside emergency department with a 2-month history of abdominal pain, back pain, and unintended weight loss. Past medical history was significant only for hypertension. Computed tomography of the abdomen and pelvis revealed scattered nodular enhancing lesions in both lobes of the liver, and subsequent magnetic resonance imaging (MRI) showed what was suspected to be extensive hepatic and bony metastatic disease. After referral to a medical oncologist, a positron emission tomography/computed tomography scan confirmed diffuse metastatic disease in the liver with subtle metastatic bone lesions. The scan did not reveal an obvious primary tumor. A subsequent liver core biopsy was positive for HMB-45 and Melan-A and negative for CK8/18, CK7, CK20, Pax-8, PSA, PSAP, chromogranin, synaptophysin, and arginase, confirming a diagnosis of metastatic melanoma. Physical examination did not reveal any skin or mucosal lesions. Due to the well-known propensity of uveal melanoma to metastasize to the liver, the patient was referred to an ophthalmologist who identified a left choroidal lesion on examination. MRI of the brain and orbits revealed an $11 \times 7 \mathrm{~mm}$ enhancing nodule along the posterolateral aspect of the left globe
Table 1. Uveal melanoma survivorship prediction

\begin{tabular}{llll}
\hline & Year 3 & Year 5 & Year 10 \\
\hline Controls* & 94 & 90 & 76 \\
Patient & $66-85$ & $44-71$ & $20-42$ \\
\hline
\end{tabular}

Estimated survival from the validated Uveal Melanoma TNM staging and survivorship algorithm, the Liverpool Uveal Melanoma Prognosticator Online (LUMPO: ocularmelanomaonline.org). Values are percentages. ${ }^{*}$ Age- and sex-matched general population control group.

(Fig. 1). There was no evidence of extraocular extension or intracranial metastasis. At this point, the patient was referred to our care.

On our examination, best-corrected visual activity was 20/20 OD and 20/50 OS. Upon further questioning, the patient reported recent changes in vision and frequent floaters in his left eye. Intraocular pressure and anterior segment findings were normal in both eyes. Fundus examination of the left eye revealed an elevated choroidal lesion with orange pigment along the superior posterior border and associated subretinal fluid (Fig. 2). B-scan ultrasonography showed a low-to-medium internal reflectivity with measurements of $8.6 \times 16.0 \times 18.1 \mathrm{~mm}$ malignant melanoma (Fig. 3 ).

Prior to evaluation by an ophthalmologist, the origin of the metastatic lesions had been unknown. Based upon the characteristic overlying orange pigment of uveal melanoma, the origin was suspected to be ocular. The patient elected to be treated with I-125 plaque brachytherapy. Fine-needle aspiration biopsy done prior to plaque placement allowed cytogenetic analysis and risk stratification, which revealed chromosome 3 loss and 8q gain, confirming the diagnosis of primary uveal melanoma.

His estimated survival from the validated Uveal Melanoma TNM staging and survivorship algorithm was calculated as 66-85 and $44-71 \%$ at 3 and 5 years, respectively (Table 1). Despite combined immunotherapy treatment with ipilimumab and nivolum$\mathrm{ab}$, our patient succumbed to his disease within 2 months of his initial presentation to the emergency department.

\section{Discussion and Conclusion}

We report a patient with symptomatic liver and bone metastasis as the presenting features of uveal melanoma. Although the risk of metastatic disease in uveal melanoma reaches $50 \%$, there is typically a latency period prior to overt metastatic disease [1]. Seventy percent of the patients are diagnosed after they present with complaints of blurred vision, visual field defects, photopsia, or irritation and pain. The majority of the remaining cases are detected incidentally on routine eye examinations [2]. Fewer than $4 \%$ of the patients have detectable metastatic disease at the time of their 
Fig. 2. Fundus examination of the left eye demonstrating an elevated choroidal lesion with orange pigment along the superior posterior border and associated subretinal fluid.

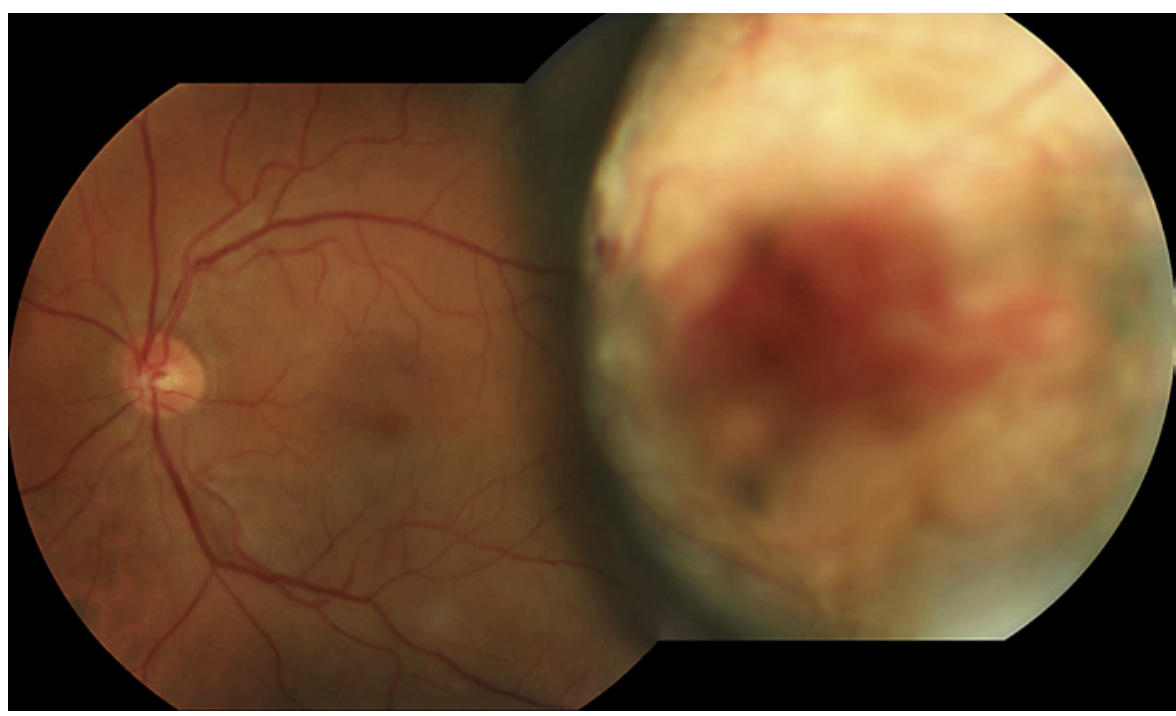

diagnosis [3]. The median time to diagnosis of systemic metastasis is 3.25 years, although several case reports describe uveal melanomas with clinical metastasis first discovered more than 20 years after initial treatment [4, 5]. The liver is the predominant site of metastasis, occurring in a reported $89 \%$ of the cases [6]. To our knowledge, this is the first report of a patient with metastatic melanoma of unknown primary ultimately diagnosed as uveal melanoma.

While histologic and cytologic evaluations are utilized in the diagnosis of most non-ocular tumors, the diagnosis of choroidal melanoma is based mainly on indirect ophthalmoscopic examination and ocular ultrasonography. Histologic and cytologic evaluation of uveal melanoma is performed for molecular prognostic testing at the time of planned treatment. In this rare case, cytogenetic findings unique to uveal melanoma provided confirmation of the clinical diagnosis of primary uveal melanoma, as monosomy 3 and trisomy 8 are features seen in uveal melanoma but not in cutaneous or mucosal melanoma $[7,8]$. Additionally, the loss of chromosome 3 and gain of chromosome 8 are associated with a very high risk of metastatic disease and death $[9,10]$.

The validated Uveal Melanoma Staging and survivorship algorithm considers demographic, histomorphological, and genetic risk factors to calculate a patient's chance of survival $[11,12]$. Of the factors considered, the one that can be influenced by clinical practice is tumor size at diagnosis. Our patient had a largest tumor diameter of 18.1 $\mathrm{mm}$, and his chance of survival was calculated as $66-85$ and $44-71 \%$ at 1 and 5 years, respectively. However, had his tumor been detected at half of that size, $9 \mathrm{~mm}$, all re-

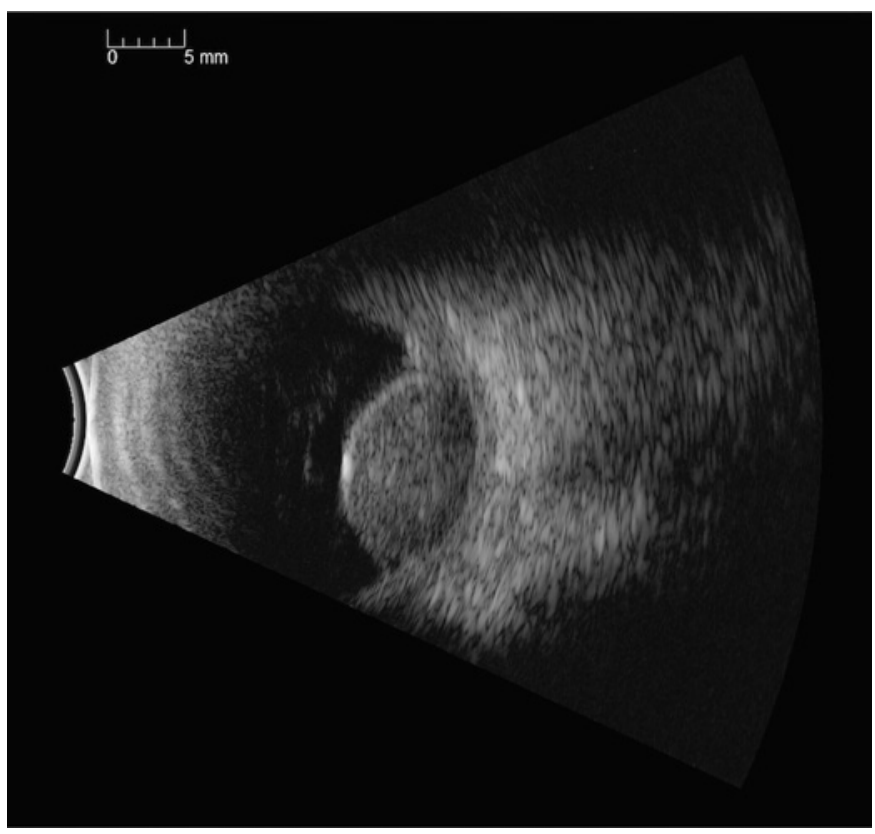

Fig. 3. B-scan ultrasound of the left eye showing low-to-medium internal reflectivity with measurements of $8.6 \times 16.0 \times 18.1 \mathrm{~mm}$ malignant melanoma.

maining factors unchanged, his chance of survival would have been calculated as $91-94$ and $80-86 \%$ at 1 and 5 years, respectively. This highlights the importance of routine comprehensive eye examinations in detecting uveal melanoma as early as possible, as tumor size is a clear prognostic factor even in those with high-risk cytogenetic findings [13]. 
Our patient was able to receive the appropriate diagnosis and therapy because medical oncology suspected a metastatic uveal melanoma despite the absence of classic ophthalmologic complaints. MRI of the brain and orbit is standard of care in staging newly discovered melanoma, and in this case, it was able to identify the ocular lesion. Although it is the most sensitive test for imaging brain metastases, it should not be used to rule out the presence of uveal melanoma. Ocular tumors $<3 \mathrm{~mm}$ in thickness are not reliably identified by MRI [14]. Because of uveal melanoma's propensity to spread hematogenously to the liver, we recommend that patients with metastatic liver melanoma of unknown origin receive a thorough ophthalmologic examination as a component of their metastatic workup.

\section{Acknowledgements}

There are no acknowledgements to make for this study.

\section{Statement of Ethics}

This report has been performed in accordance with the Declaration of Helsinki.

\section{Disclosure Statement}

We wish to confirm that there are no known conflicts of interest associated with this publication, and there has been no significant financial or proprietary support for this work that could have influenced its outcome.

\section{Funding Sources}

There are no funding sources for this study.

\section{Author Contributions}

All authors are responsible for contributing to the design or interpretation of the work, drafting the work and revising it critically, approving the final version of the work to be published, and agreeing to be accountable for all aspects of the work.

\section{References}

1 Eskelin S, Pyrhönen S, Summanen P, HahkaKemppinen M, Kivelä T. Tumor doubling times in metastatic malignant melanoma of the uvea: tumor progression before and after treatment. Ophthalmology. 2000 Aug;107(8): 1443-9.

2 Jovanovic P, Mihajlovic M, Djordjevic-Jocic J, Vlajkovic S, Cekic S, Stefanovic V. Ocular melanoma: an overview of the current status. Int J Clin Exp Pathol. 2013 Jun;6(7):1230-44.

3 Finger PT, Kurli M, Reddy S, Tena LB, Pavlick AC. Whole body PET/CT for initial staging of choroidal melanoma. Br J Ophthalmol. 2005 Oct;89(10):1270-4.

4 Kolandjian NA, Wei C, Patel SP, Richard JL, Dett T, Papadopoulos NE, et al. Delayed systemic recurrence of uveal melanoma. Am J Clin Oncol. 2013 Oct;36(5):443-9.

5 Kujala E, Mäkitie T, Kivelä T. Very long-term prognosis of patients with malignant uveal melanoma. Invest Ophthalmol Vis Sci. 2003 Nov;44(11):4651-9.
6 Diener-West M, Reynolds SM, Agugliaro DJ Caldwell R, Cumming K, Earle JD, et al. Development of metastatic disease after enrollment in the COMS trials for treatment of choroidal melanoma: Collaborative Ocular Melanoma Study Group Report No. 26. Arch Ophthalmol. 2005 Dec;123(12):1639-43.

7 Horsman DE, Sroka H, Rootman J, White VA. Monosomy 3 and isochromosome $8 \mathrm{q}$ in a uveal melanoma. Cancer Genet Cytogenet. 1990 Apr;45(2):249-53.

8 Sisley K, Rennie IG, Cottam DW, Potter AM, Potter CW, Rees RC. Cytogenetic findings in six posterior uveal melanomas: involvement of chromosomes 3, 6, and 8. Genes Chromosomes Cancer. 1990 Sep;2(3):205-9.

9 Aalto Y, Eriksson L, Seregard S, Larsson O, Knuutila S. Concomitant loss of chromosome 3 and whole arm losses and gains of chromosome 1,6 , or 8 in metastasizing primary uveal melanoma. Invest Ophthalmol Vis Sci. 2001 Feb;42(2):313-7.

10 Prescher G, Bornfeld N, Hirche H, Horsthemke B, Jöckel KH, Becher R. Prognostic implications of monosomy 3 in uveal melanoma. Lancet. 1996 May;347(9010):1222-5.
11 DeParis SW, Taktak A, Eleuteri A, Enanoria W, Heimann H, Coupland SE, et al. External validation of the Liverpool uveal melanoma prognosticator online. Invest Ophthalmol Vis Sci. 2016 Nov;57(14):6116-22

12 Eleuteri A, Damato B, Coupland SE, Taktak A. Enhancing survival prognostication in patients with choroidal melanoma by integrating pathologic clinical and genetic predictors of metastases. Int J Biomed Eng Technol. 2012;8(1):18-35.

13 Shields CL, Furuta M, Thangappan A, Nagori S, Mashayekhi A, Lally DR, et al. Metastasis of uveal melanoma millimeter-by-millimeter in 8033 consecutive eyes. Arch Ophthalmol. 2009 Aug;127(8):989-98.

14 Mafee MF, Peyman GA, Peace JH, Cohen SB, Mitchell MW. Magnetic resonance imaging in the evaluation and differentiation of uveal melanoma. Ophthalmology. 1987 Apr;94(4): 341-8. 